\title{
Blocking Interleukin-6 and Interleukin-8 Signaling Inhibits Cell Viability, Colony-forming Activity, and Cell Migration in Human Triple-negative Breast Cancer and Pancreatic Cancer Cells
}

\author{
SHENGLING FU ${ }^{1,2}$ and JIAYUH LIN ${ }^{2}$ \\ ${ }^{1}$ Department of Thoracic Surgery, Tongji Hospital, Tongji Medical College, \\ Huazhong University of Science and Technology, Wuhan, P.R. China; \\ ${ }^{2}$ Department of Biochemistry and Molecular Biology, School of Medicine, \\ University of Maryland, Baltimore, MD, U.S.A.
}

\begin{abstract}
Background/Aim: Interleukin-6 (IL-6) and interleukin-8 (IL-8) play important roles in the progression of triple-negative breast cancer (TNBC) and pancreatic ductal adenocarcinoma (PDAC). This is the first experiment to combine small molecules targeting these two signaling pathways to treat TNBC and PDAC cells. Materials and Methods: Cell viability, colony formation and cell migration assays were conducted when TNBC or PDAC cells were treated with bazedoxifene (targeting IL-6) or reparixin/SCH527123 (targeting IL-8) or their combination. Results: The combined treatment had a more potent inhibition of cell viability, colony formation and cell migration than monotherapy in TNBC and PDAC cells. The results also showed that the combination of bazedoxifene with SCH527123 seemed to be more effective than that with reparixin in inhibiting cell viability and colony formation of TNBC. Conclusion: Novel drug combinations of bazedoxifene and reparixin, as well as bazedoxifene and SCH527123 may provide more effective treatments for TNBC and PDAC.
\end{abstract}

Breast cancer is the most predominant cancer in women worldwide $(1,2)$. Triple-negative breast cancer (TNBC) accounts for $15-20 \%$ of all cases of breast cancer. It is more aggressive than other types of breast cancer and has a worse prognosis, with a high recurrence rate within the first 3 years after diagnosis (3). In the absence of new effective targeted

Correspondence to: J. Lin, Department of Biochemistry and Molecular Biology, University of Maryland Marlene and Stewart Greenebaum Comprehensive Cancer Center, University of Maryland School of Medicine, 108 N. Greene Street, Baltimore, MD, 21201, U.S.A. E-mail: JLin@ som.umaryland.edu

Key Words: Interleukin-6, interleukin-8, breast cancer, pancreatic cancer, bazedoxifene. therapies, conventional chemotherapy is still one of the main treatment options for TNBC, however, the side-effects of chemotherapeutics are serious. The treatment of TNBC is still a challenge (4).

Pancreatic ductal adenocarcinoma (PDAC) accounts for $>90 \%$ pancreatic cancer and has the lowest survival rate among common cancers $(1,2)$. Contrary to the steady increase in survival observed in most types of cancer, little progress has been made for pancreatic cancer, and most patients are diagnosed with disease at advanced stage with a 5-year survival rate of only $3 \%$ (1). At present, there is no effective drug or treatment options for pancreatic cancer, development of more effective drugs is of the utmost urgency.

The growth of TNBC requires the simultaneous autocrine expression of interleukin 6 (IL-6) and interleukin 8 (IL-8), and their co-expression was found to be essential for the growth of xenograft tumors, while inhibiting their expression significantly suppressed cell survival and colony formation in vitro, as well as growth of xenograft tumors in vivo (5). In addition, resistant breast cancer cell lines secrete more IL-6 and IL-8 than sensitive breast cancer cell lines. Neutralizing antibodies against IL-6 and IL-8 partially reversed the resistance of breast cancer to chemotherapeutic drugs. The inhibition of the expression of endogenous IL- 6 and IL- 8 by siRNA technology also significantly enhanced the drug sensitivity of resistant breast cancer cells (6). These novel findings demonstrate the necessity and potential of combined inhibition of IL-6 and IL-8 for the treatment of TNBC. Clinical studies have shown that both IL-6 and IL-8 are overexpressed in pancreatic cancer tissues and blood circulation, and both promote the occurrence, metastasis and malignant progression of pancreatic cancer and are associated with poor prognosis (7-16). However, currently there are no experimental or clinical studies about joint suppression of IL6 and IL- 8 in the treatment of pancreatic cancer. Combined 
inhibition of IL-6 and IL-8 may be a novel and effective treatment strategy for the treatment of TNBC and PDAC.

The Food and Drug Administration-approved drug bazedoxifene is a third-generation selective estrogen receptor modulator for the prevention and treatment of osteoporosis in postmenopausal women and has been shown to be relatively safe and well tolerated (17). We discovered bazedoxifene to be a novel inhibitor of IL-6/ glycoprotein 130 (GP130) protein-protein interactions and it inhibited PDAC and TNBC cells (18).

As a small-molecule drug against IL-8, reparixin was originally used clinically to reduce ischemia-reperfusion injury (19), but it was later discovered to block C-X-C chemokine receptor 1 (CXCR1) and $\mathrm{C}-\mathrm{X}-\mathrm{C}$ chemokine receptor 2 (CXCR2) signaling pathway in many cancer types such as breast and thyroid cancer $(20,21)$. Another IL-8CXCR1/2 small-molecule inhibitor, SCH527123, showed antitumor effects against many tumor types such as melanoma (22), and breast (23) and colon (24) cancer. We found that reparixin and SCH527123 were effective at inhibiting pancreatic cancer neoplastic features such as cell viability, proliferation, colony formation, and migratory potential in vitro laboratory culture system (25).

The objective of this study was to assess the efficacy of bazedoxifene combined with reparixin or SCH527123 on cell viability, cell migration and colony formation of TNBC and PDAC cells

\section{Materials and Methods}

Cell culture and reagents. SUM159 TNBC cells were obtained from Dr. Max S. Wicha (University of Michigan, Ann Arbor, MI), MDAMB-231 TNBC cells, HPAC and Capan-1 PDAC cells were purchased from the American Type Culture Collection (Manassas, VA, USA). All cell lines were routinely cultured in Dulbecco's modified Eagle's medium with $4.5 \mathrm{~g} / \mathrm{l} \mathrm{L-glutamine}$ and sodium pyruvate (DMEM; Mediatech Inc., Manassas, VA, USA) supplemented with $10 \%$ fetal bovine serum (FBS; Atlanta Biologicals, Flowery Branch, GA, USA), and $1 \%$ penicillin/ streptomycin (Sigma-Aldrich, Merck KGaA, Darmstadt, Germany) and then grown in a humidified $37^{\circ} \mathrm{C}$ incubator with $5 \% \mathrm{CO}_{2}$. Routinely, they were assessed microscopically for the expected morphologies.

Reagents in the study were as follows: Bazedoxifene acetate was purchased from Sigma-Aldrich (Merck $\mathrm{KGaA}$ ), reparixin was obtained from INDOFINE Chemical Company (Hillsborough, NJ, USA), SCH527123 was bought from AdooQ BioScience (Irvine, CA, USA). These drugs were dissolved in sterile dimethyl sulfoxide (DMSO; Sigma-Aldrich, Merck KGaA) to make a $20 \mathrm{mM}$ stock solution and they were stored at $-20^{\circ} \mathrm{C}$. 3-(4,5-dimethylthiazol-2yl)-2,5-diphenyltetrazolium bromide and crystal violet were bought from Sigma-Aldrich (Merck KGaA) and $N, N$-dimethylformamide (DMF) was obtained from Fisher Scientific (Waltham, MA, USA).

MTT assay. MDA-MB-231, SUM159, HPAC and Capan-1 were seeded in 96-well microtiter plates at a density of 3,000 cells per well and cultured in $100 \mu \mathrm{l}$ medium at $37^{\circ} \mathrm{C}$. The next day, the culture medium in each well was not only maintained at $100 \mu$ as the final volume, but also supplemented with bazedoxifene ( 5 or $10 \mu \mathrm{mol} / \mathrm{l})$ or reparixin/SCH527123 (40 or $60 \mu \mathrm{mol} / \mathrm{l}$ ), their combination or vehicle control (DMSO) at $37^{\circ} \mathrm{C}$. Forty-eight hours later, $25 \mu \mathrm{l}$ of MTT was added to each well. After an incubation for $4 \mathrm{~h}$ at $37^{\circ} \mathrm{C}$, each sample was supplemented with $150 \mu$ l of DMF solubilization solution followed by an incubation overnight in a sealed moistened chamber protected from light at room temperature. The cell viability was assessed using, the absorbance at $595 \mathrm{~nm}$ in each sample, reflecting the amount of MTT taken by the metabolically active cells, was compared to that in the mock control wells (treated with DMSO) and then quantified. The untreated cells were set at $100 \%$ and the cell viability of drug-treated cells was determined relative to untreated cells. Synergistic, additive, or antagonistic effects of bazedoxifene and reparixin/SCH527123 treatment were determined based on the theorem of Chou and Talalay (26), and the combination index (CI) was determined using data obtained from the MTT assay with CompuSyn software (www.combosyn.com). The CI values indicate a synergistic effect when $<1$, an antagonistic effect when $>1$, and an additive effect when equal to 1 .

Colony-formation assay. The experiment was carried out according to the method described previously (25). MDA-MB-231, SUM159, HPAC and Capan- 1 cells were seeded in 6-well plates at a density of 1,000 cells per well and incubated at $37^{\circ} \mathrm{C}$ overnight. The next day, cells were incubated with vehicle DMSO, or bazedoxifene $(2.5$ or $5 \mu \mathrm{mol} / \mathrm{l}$ ) or reparixin/SCH527123 (40 or $60 \mu \mathrm{mol} / \mathrm{l}$ ) or the combination at $37^{\circ} \mathrm{C}$. At 3-day intervals, cells were replenished with fresh growth medium containing the same drug. In 7 days, the medium was changed with fresh medium without drugs and the medium was changed every week. After 2 weeks, the cells were washed twice with PBS and fixed with cold methanol for $30 \mathrm{~min}$ at $4^{\circ} \mathrm{C}$ followed by a staining with $1 \%$ crystal violet dye (dissolved in $25 \%$ methanol) at room temperature for $1 \mathrm{~h}$. The plates were washed with distilled water, dried and scanned with Epson Perfection V550 Photo Color Scanner

Wound-healing assay. SUM159, MDA-MB-231 and HPAC cells were seeded in six-well plates and incubated at $37^{\circ} \mathrm{C}$ overnight. The next day when cells reached $100 \%$ confluent, the monolayer was scratched by using a $100-\mu l$ pipette tip. After washing with PBS twice, cells were treated with DMSO, or bazedoxifene $(5$ or $10 \mu \mathrm{mol} / \mathrm{l})$ or reparixin/SCH527123 (40 or $60 \mu \mathrm{mol} / \mathrm{l}$ ) or bazedoxifene plus reparixin/SCH527123. Cells were incubated for $17-30 \mathrm{~h}$ at $37^{\circ} \mathrm{C}$ and observed under a microscope, methods for photographing and calculating cell mobility were as described in the literature (25); the CI value was calculated in the same way as described above.

Statistical analysis. Significance of correlations was determined using GraphPad Prism 5 software (GraphPad Software Inc., San Diego, CA, USA). Differences were analyzed with one-way ANOVA and Tukey's multiple comparison test for multiple comparisons. Data are presented as mean \pm standard error, and significance was set at $p<0.05$.

\section{Results}

Bazedoxifene combined with reparixin or SCH527123 synergistically inhibited cell viability of TNBC and PDAC cells. To investigate whether bazedoxifene plus reparixin/SCH527123 can generate more potent suppression 
A

MDA-MB-231

$\mathrm{Cl}=0.80122$

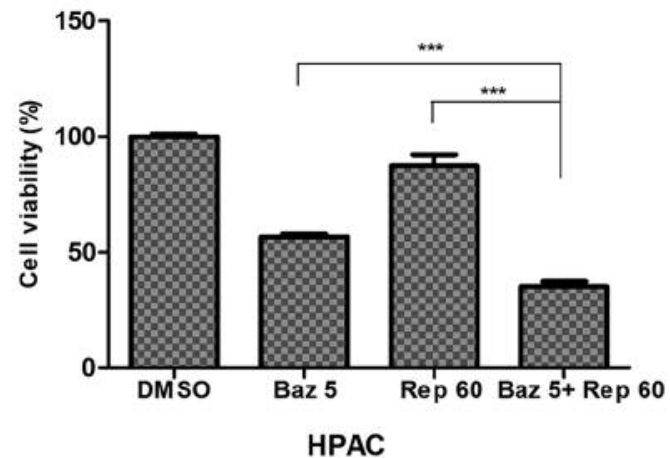

HPAC

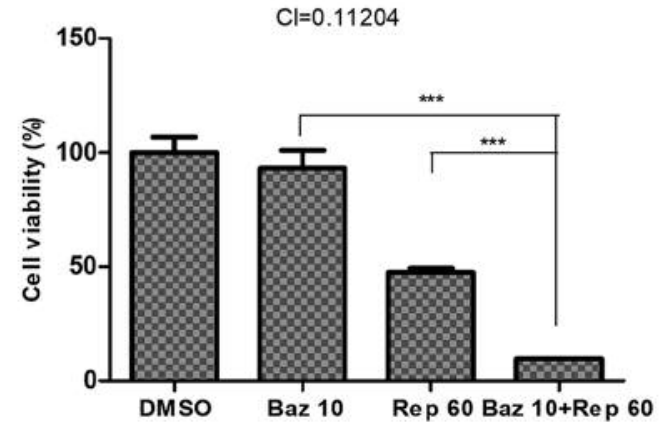

B
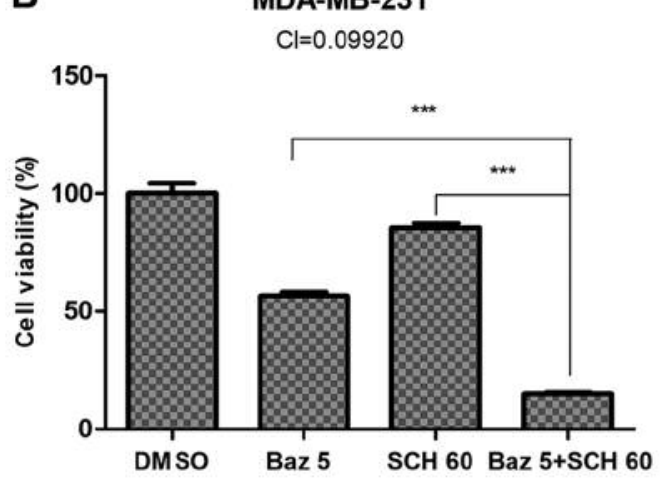

HPAC

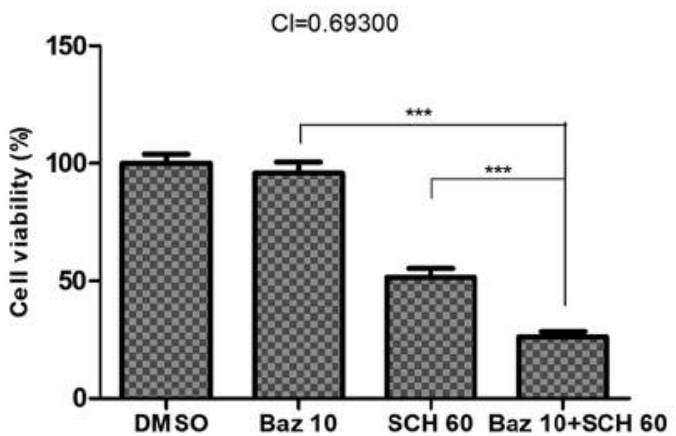

SUM159

$\mathrm{Cl}=0.70122$

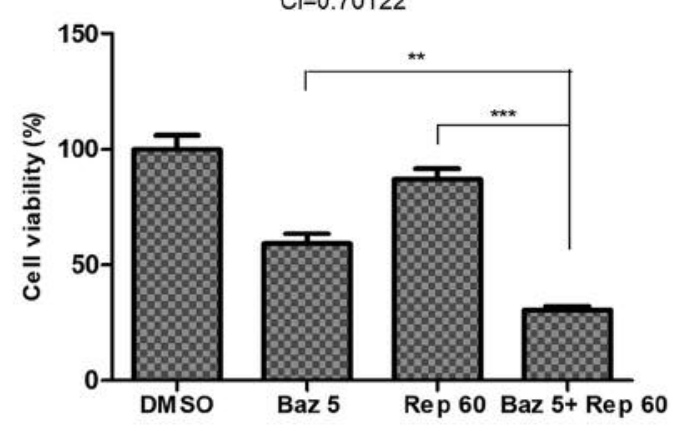

Capan-1

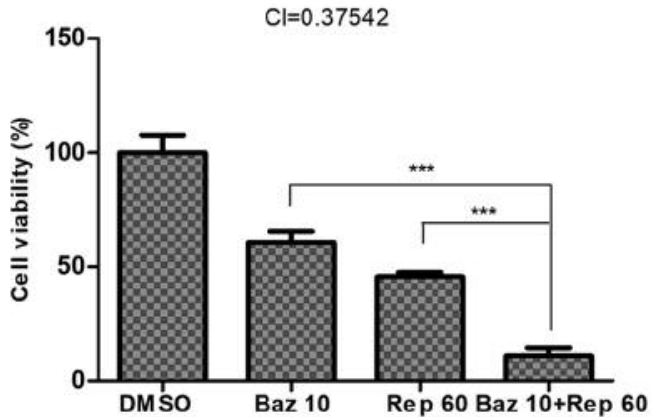

SUM159

$\mathrm{Cl}=0.10235$

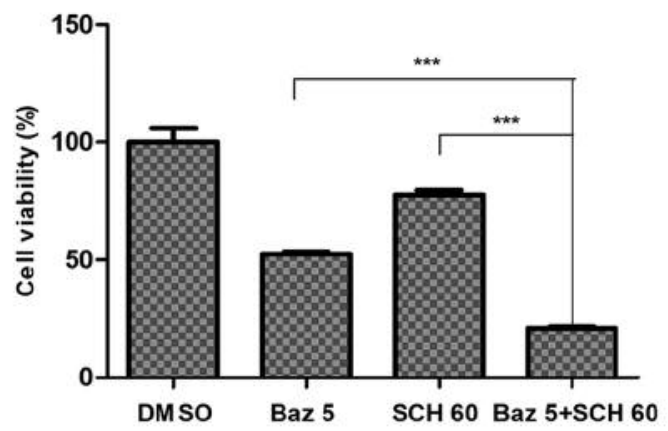

Capan-1

$\mathrm{Cl}=0.24581$

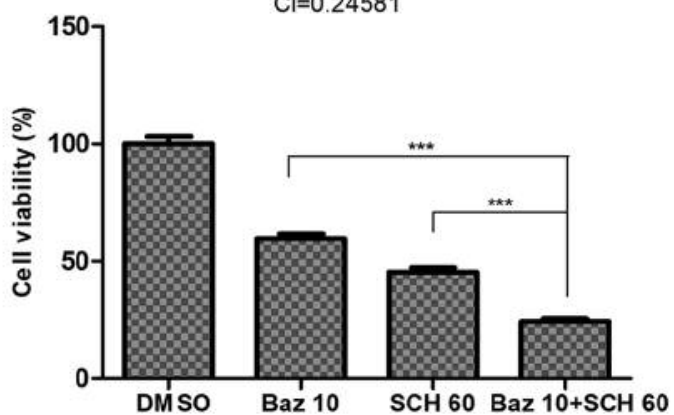

Figure 1. Bazedoxifene (Baz) combined with reparixin (Rep) or SCH527123 (SCH) synergistically inhibited cell viability of triple-negative breast cancer and pancreatic ductal adenocarcinoma cells. MDA-MB-231, SUM159, HPAC and Capan-1 cells were seeded in 96-well plate at a density of 3,000 cells per well. Cells were treated with bazedoxifene (5 or $10 \mu \mathrm{mol} / \mathrm{l})$ or reparixin (40 or $60 \mu \mathrm{mol} / \mathrm{l})$ (A) or SCH527123 (SCH; 40 or 60 $\mu \mathrm{mol} / \mathrm{l})(B)$ or their combination or dimethyl sulfoxide (DMSO) in triplicate for $48 \mathrm{~h}$ and cell viability was tested by MTT assay. Significantly different at $* * p<0.01$ and $* * * p<0.001$. 
A

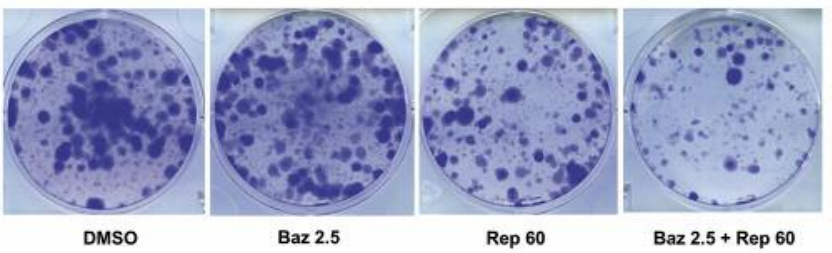

MDA-MB-231
HAPC
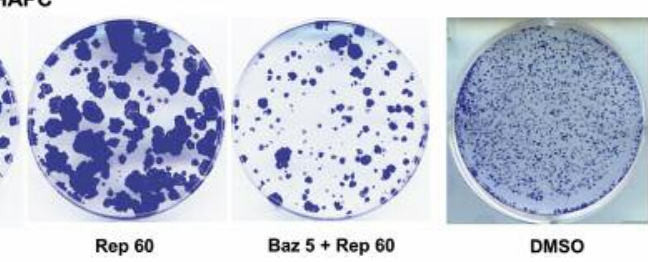

SUM159

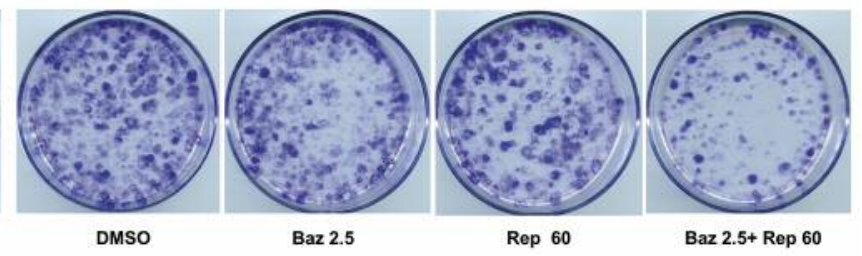

Capan-1

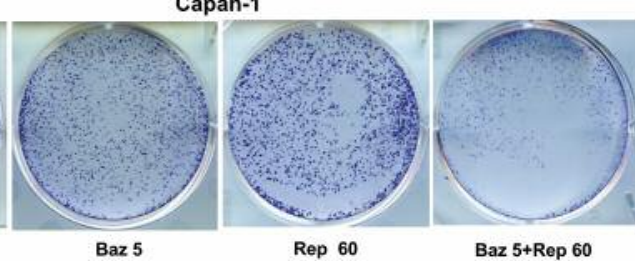

B

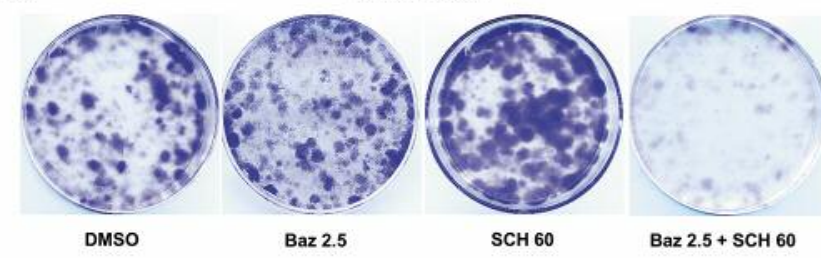

MDA-MB-231
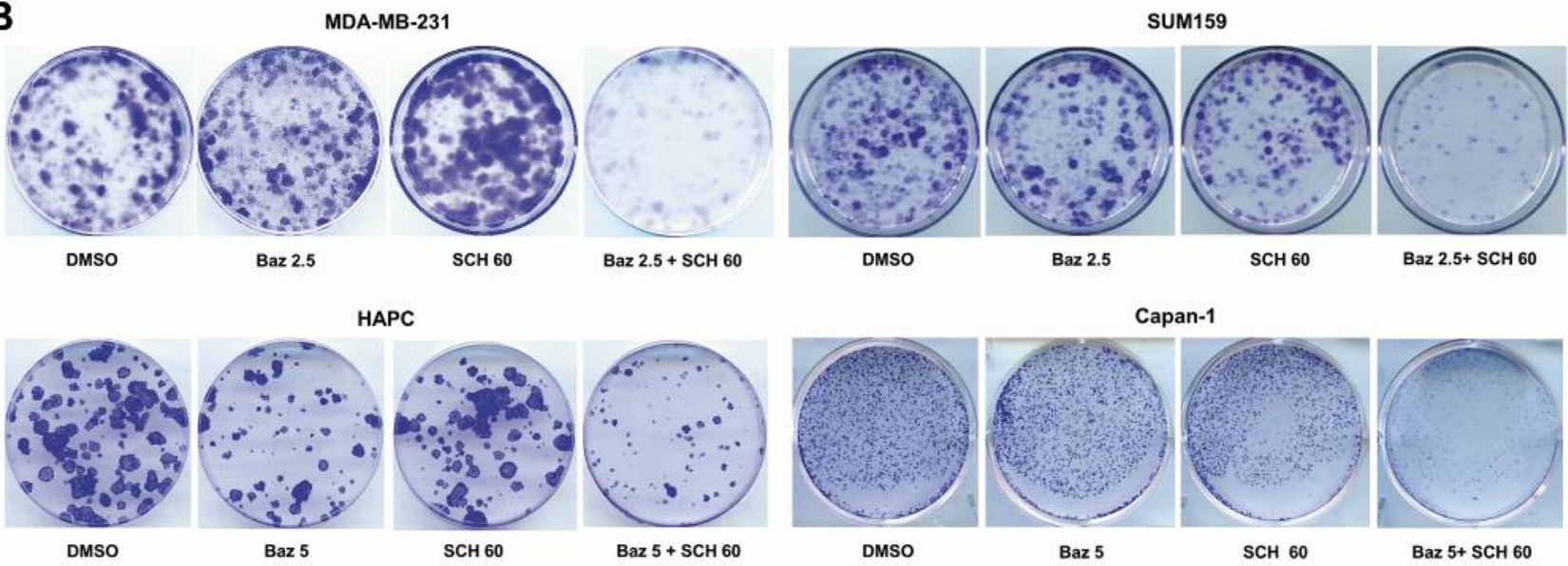

HAPC

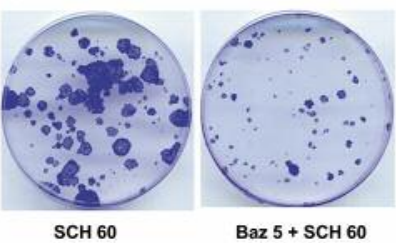

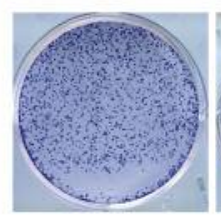

DMSO

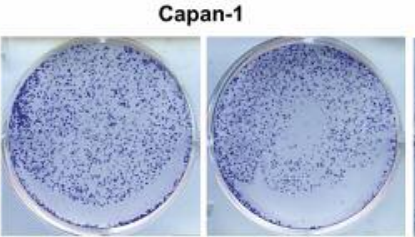

SCH 60

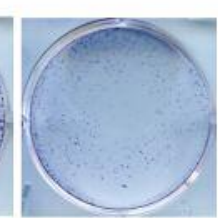

Baz 5+ SCH 60

Figure 2. Combined treatment of bazedoxifene (Baz) with reparixin (Rep) or SCH527123 (SCH) generated more potent inhibitory effects on colonyforming activity of triple-negative breast cancer and pancreatic ductal adenocarcinoma cells than either drug alone. MDA-MB-231, SUM159, HPAC and Capan-1 cells were seeded in 6-well plates at a density of 1,000 cells per well. The next day, cells were incubated with vehicle dimethyl sulfoxide (DMSO), or bazedoxifene (Baz; 2.5 or $5 \mu \mathrm{mol} / \mathrm{l})$ or reparixin (Rep; 40 or $60 \mu \mathrm{mol} / \mathrm{l})$ (A) or SCH527123 (SCH; 40 or $60 \mu \mathrm{mol} / \mathrm{l})$ (B) or their combination. At 3-day intervals, cells were replenished with fresh growth medium containing the same drug. In 7 days, the medium was changed with fresh medium without drugs and the medium was changed every week.

of cell viability of TNBC and PDAC, MDA-MB-231, SUM159, HPAC and Capan-1 cells were treated with different concentrations of bazedoxifene $(5$ or $10 \mu \mathrm{mol} / 1)$ or reparixin/SCH527123 (40 or $60 \mu \mathrm{mol} / 1)$ or their combination. After 48-hour treatment, greater inhibition of cell viability was seen with the combination treatment of bazedoxifene with reparixin (Figure 1A) and SCH527123 (Figure 1B) compared to monotherapy. The CI values of all the combination treatments were less than 1, suggesting there was synergism in the combination treatments of bazedoxifene with reparixin and SCH527123. Bazedoxifene with SCH527123 seemed to inhibit cell viability more significantly than bazedoxifene with reparixin in two TNBC cell lines tested, and bazedoxifene with reparixin seemed to inhibit cell viability more significantly than bazedoxifene with SCH527123 in two PDAC cell lines.

Combination treatment of bazedoxifene with reparixin or SCH527123 generated greater potent inhibitory effects on colony-forming activity of TNBC and PDAC cells than either drug alone. We next evaluated the effect of bazedoxifene combined with reparixin or SCH527123 on colony formation. MDA-MB-231, SUM159, HPAC and Capan-1 cells, seeded in six-well plates with 1,000 cells per well, were treated with different concentrations of bazedoxifene ( 2.5 or $5 \mu \mathrm{mol} / \mathrm{I}$ ) or reparixin/SCH527123 (40 or $60 \mu \mathrm{mol} / \mathrm{I}$ ) or their combination. Representative images are presented in Figure 2A (bazedoxifene combined with reparixin) and 
A
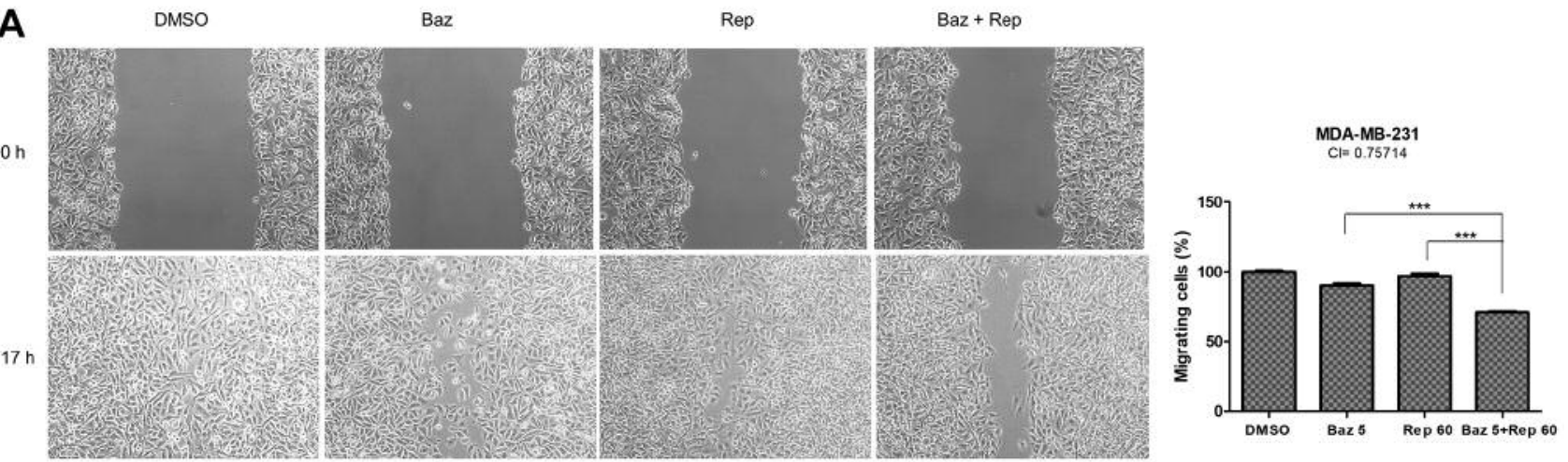

B

DMSO

Baz

$\mathrm{SCH}$

$\mathrm{Baz}+\mathrm{SCH}$
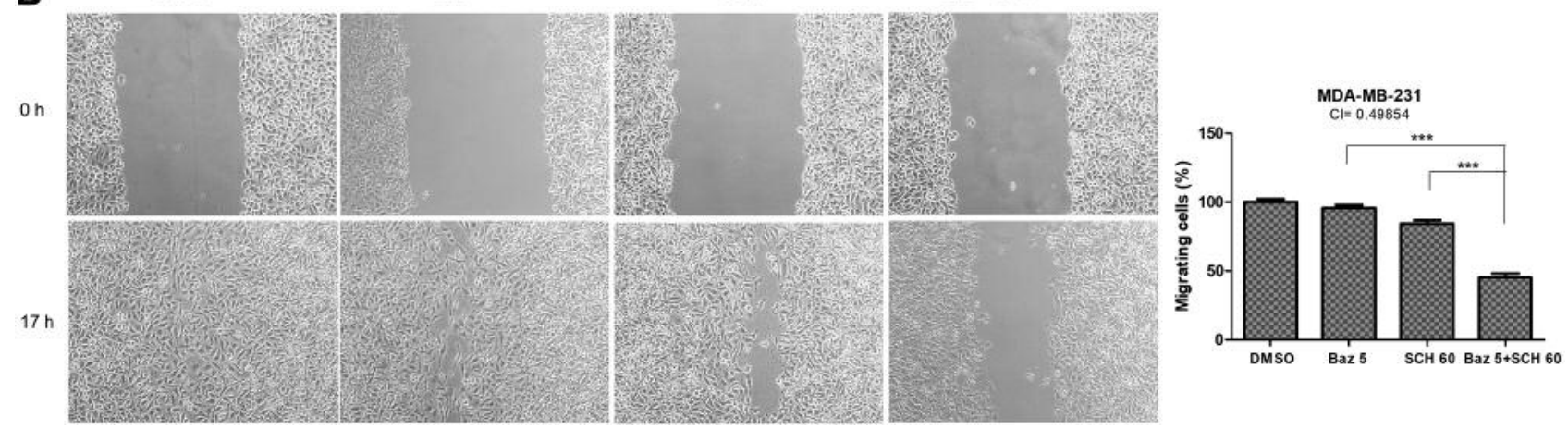

Figure 3. Bazedoxifene (Baz) combined with reparixin (Rep) or SCH527123 (SCH) synergistically inhibited cell migration of MDA-MB-231C cells. MDA-MB-231 cells were seeded in a 6-well plate, then a wound-healing assay for migration was carried out by scratching the cells with 100- $\mu$ l tip and cells were treated with vehicle dimethyl sulfoxide (DMSO), or bazedoxifene (Baz; 5 or $10 \mu$ mol/I) or reparixin (Rep; 40 or $60 \mu$ mol/I) (A) or SCH527123 (SCH; 40 or $60 \mu \mathrm{mol} / \mathrm{l})(\mathrm{B})$ or their combination. Magnification, $\times 100$. The CI values of all the combination treatments were less than 1. ***Significantly different at $p<0.001$.

Figure 2B (bazedoxifene combined with SCH527123). Consistent with these findings, Figure 2 shows that colony formation was inhibited more obviously under the combined treatment of bazedoxifene with reparixin or SCH527123 than under monotherapy. It seemed that bazedoxifene/SCH527123 inhibited colony formation more significantly than bazedoxifene with reparixin in the two TNBC cell lines tested, and the inhibition of the two combinations on colony formation by PDAC cells appeared to be similar and both combinations had a good inhibitory effect.

Bazedoxifene combined with reparixin or SCH527123 synergistically inhibited cell migration of TNBC and PDAC cells. Simultaneous inhibition of IL-6/IL-8 receptors reduced metastasis in a mouse xenograft model (27), therefore combined suppression of IL-6/IL-8 might generate greater inhibition of cancer cell migration. Therefore, we next evaluated the effect of bazedoxifene combined with reparixin or SCH527123 on cell migration which is an important process in tumor invasion and metastasis. Representative images are shown in Figures 3-5, combined therapy showed more significant inhibitory effects of cell migration on MDAMB-231 (Figure 3), SUM159 (Figure 4) and HAPC (Figure 5 ) cells relative to monotherapy. These results suggested that simultaneous inhibition of IL-6/IL- 8 by combined treatment of bazedoxifene with reparixin or SCH527123 block the cells migration of TNBC cells and PDAC cells, the CI values of all the combination treatments were less than 1, suggesting there was synergism in the combination treatments of bazedoxifene with reparixin or SCH527123.

\section{Discussion}

For the first time, the small molecules bazedoxifene, reparixin and SCH527123, which have been clinically validated for safety, were used to investigate the effect of a combination of bazedoxifene with reparixin or SCH52712 against IL-6/IL-8 signaling pathways to inhibit TNBC. The results showed that the combination treatment synergistically inhibited the cell viability and cell migration of TNBC, more potent inhibition of colony formation was also achieved. The effect of combined inhibition of IL-6/IL-8 signaling pathway in PDAC 
A

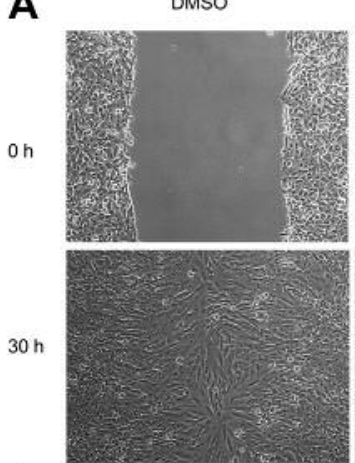

B
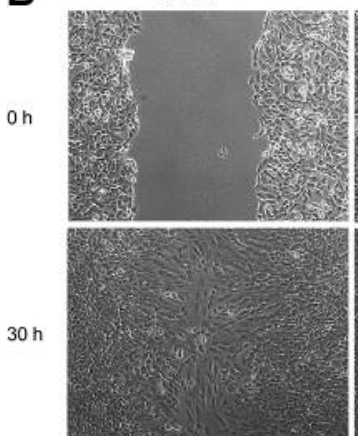

Baz

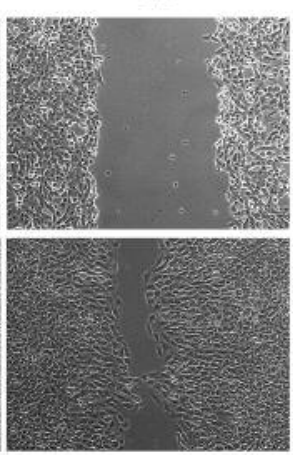

Baz
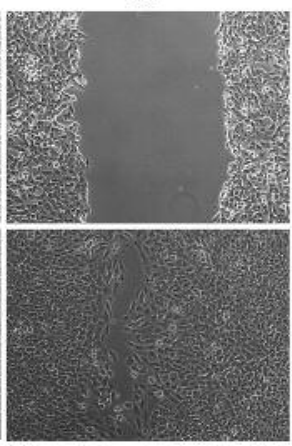

Rep

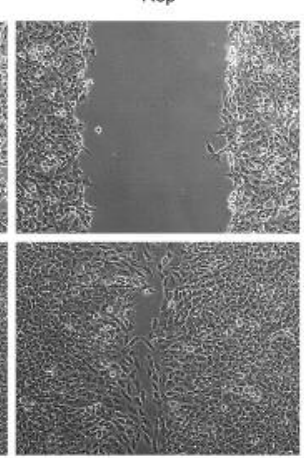

$\mathrm{SCH}$
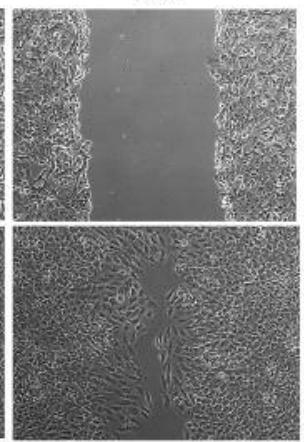

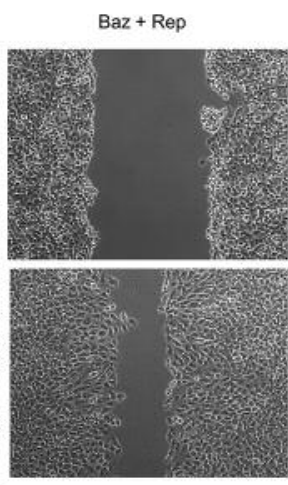

$\mathrm{Baz}+\mathrm{SCH}$

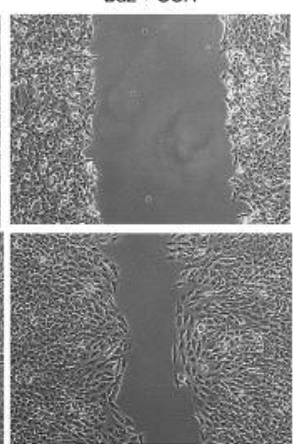

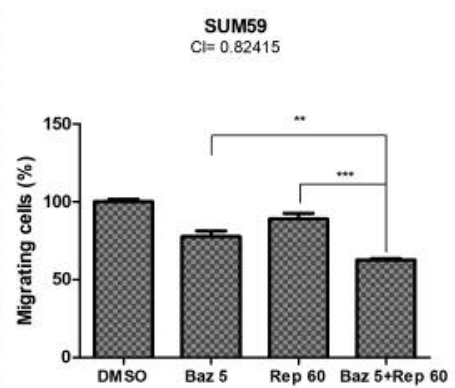

Sum59

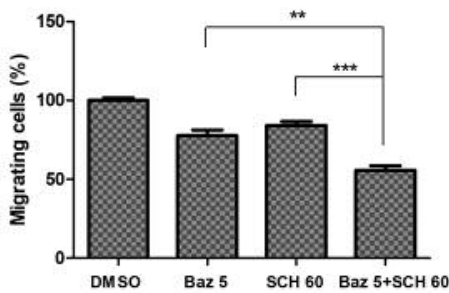

Figure 4. Bazedoxifene (Baz) combined with reparixin (Rep) or SCH527123 (SCH) synergistically inhibited cell migration of SUM159 cells. SUM159 cells were seeded in 6-well plates, then a wound-healing assay for migration was carried out by scratching the cells with 100- $\mu$ lip and cells were treated with vehicle dimethyl sulfoxide (DMSO), or bazedoxifene (Baz; 5 or $10 \mu \mathrm{mol} / \mathrm{I}$ ) or reparixin (Rep; 40 or $60 \mu \mathrm{mol} / \mathrm{I})$ (A) or SCH527123 $(\mathrm{SCH} ; 40$ or $60 \mu \mathrm{mol} / \mathrm{l})(\mathrm{B})$ or their combination. Magnification, $\times 100$. The CI values of all the combination treatments were less than 1. Significantly different at ${ }^{* *} p<0.01$ and $* * * p<0.001$.

was also investigated for the first time to our knowledge. The results showed that combination therapy (bazedoxifene with reparixin or SCH527123) synergistically inhibited cell viability and cell migration of PDAC cells, and inhibited colony formation better than either drug alone. The results also showed that the combination of bazedoxifene and SCH527123 seemed to be more effective than the combination of bazedoxifene and reparixin to inhibit cell viability and colony formation of TNBC.

Evidence indicates that overexpression of IL-6 and dysregulation of IL-6 signaling pathway play key roles in the development and metastasis of many cancer types, including pancreatic and breast cancer $(10,11,28)$. TNBC cells secrete the highest levels of IL-6 in contrast to other types of breast cancer, and autocrine IL-6 promotes TNBC growth (5). Studies have shown that gene silencing of IL-6 expression can inhibit the formation of colonies and cell survival of TNBC cells in vitro, as well as xenograft tumor growth in vivo (5). The autocrine production of IL-6 by tumor cells also promotes multidrug resistance (29), and using different methods to inhibit the expression of IL-6 may enhance the sensitivity of tumor to chemotherapeutics and reduce multidrug resistance. Due to limited treatment options for TNBC, IL-6 signaling pathway inhibitors may provide important new therapeutic options.

Our previous studies showed bazedoxifene may be a potential therapeutic agent for human pancreatic cancer, rhabdomyosarcoma and medulloblastoma (30-32). Bazedoxifene was studied in estrogen receptor-positive breast cancer $(33,34)$, however, there is little research about the application of bazedoxifene in the treatment of TNBC.

There is growing evidence that IL- 8 and $\mathrm{C}-\mathrm{X}-\mathrm{C}$ chemokine receptor $1 / 2(\mathrm{CXCR} 1 / 2)$ play a key role in the initiation and progression of various tumor types, including pancreatic and breast cancer $(9,13-16,35)$. Most cancer cells, including TNBC and pancreatic cancer express high levels of IL-8 (9, $13,14)$. Targeting IL-8 signaling has proven efficacious in vivo models of breast cancer (36). Small molecule inhibitors such as reparixin and SCH527123 are potent inhibitors of CXCR1/2. They have been used in clinical trials to suppress the progression of breast, colon and colorectal cancer $(20,24$, 37). However, clinical trials of reparixin and SCH527123 for the treatment of breast cancer, ischemia-reperfusion injury and asthma showed noticeable cytotoxicity $(19,37,38)$. 
A

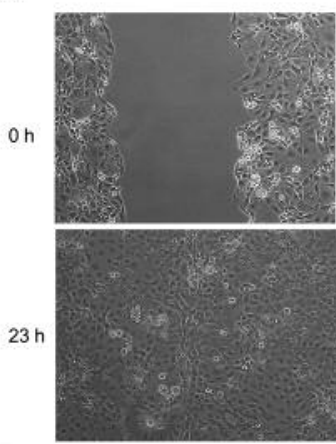

B

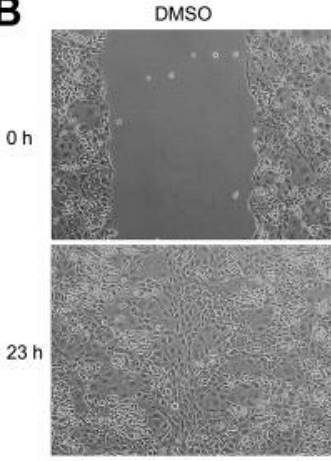

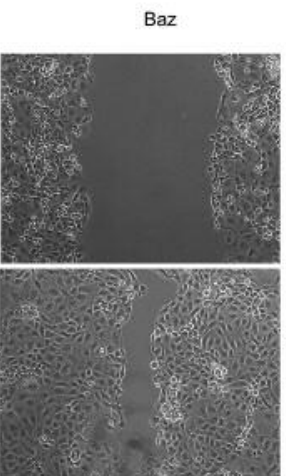

Baz

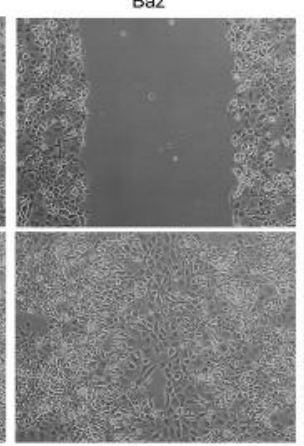

Rep

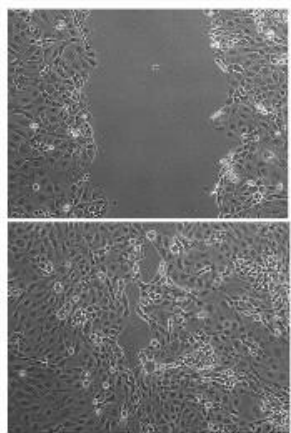

$\mathrm{SCH}$

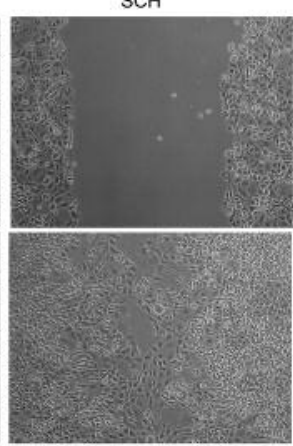

$\mathrm{Baz}+\mathrm{Rep}$

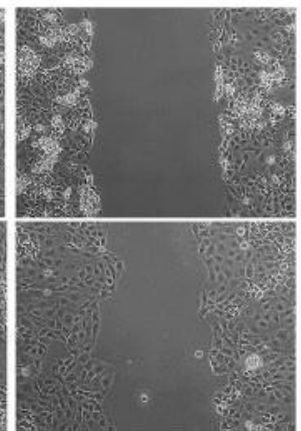

$\mathrm{Baz}+\mathrm{SCH}$

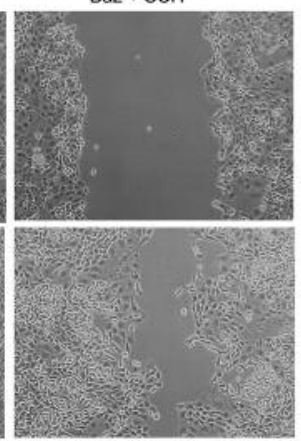

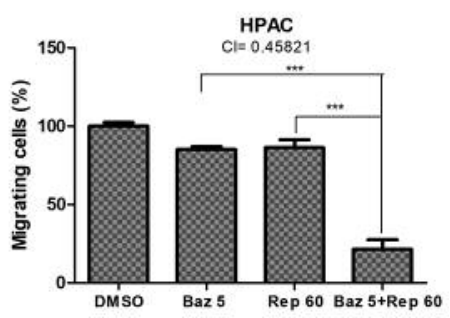

HPAC $\mathrm{Cl}=0.52549$

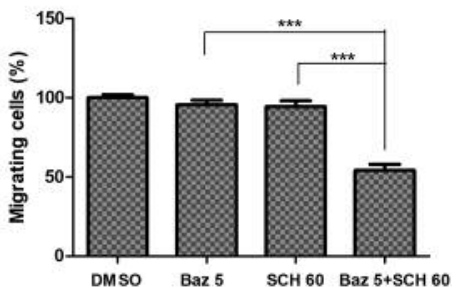

Figure 5. Bazedoxifene (Baz) combined with reparixin (Rep) or SCH527123 (SCH) synergistically inhibited cell migration of HPAC cells. HPAC

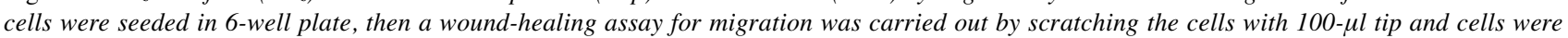
treated with vehicle dimethyl sulfoxide (DMSO), or bazedoxifene (Baz; 5 or $10 \mu \mathrm{mol} / \mathrm{I})$ or reparixin (Rep; 40 or $60 \mu \mathrm{mol} / \mathrm{I})$ (A) or SCH527123 $(\mathrm{SCH} ; 40$ or $60 \mu \mathrm{mol} / \mathrm{l})(\mathrm{B})$ or their combination. Magnification, $\times 100$. The CI values of all the combination treatments were less than 1. $* * *$ Significantly different at $p<0.001$.

It is important to note that combined inhibition of IL-6 and IL-8 signaling pathway is of great importance for the treatment of TNBC $(5,39)$. The joint inhibition of IL-6 and IL-8 expression or of their receptors caused more pronounced inhibition of TNBC colony formation than either of the cytokines or receptors alone (5). Elevated expression of IL-6 and IL-8 may be associated with multidrug resistance in human breast cancer cells, whereas pretreatment of mesenchymal stem cells with cisplatin resulted in secretion of IL-6 and IL-8, and this resulted in increased chemoresistance in breast cancer cells (40). These data together suggest that joint inhibition of IL- 6 and IL- 8 is required for the treatment of TNBC.

There have been several studies on the combination of targeting IL-6 and IL-8 signaling pathways in the treatment of breast cancer $(5,6,39)$. However, no similar study was found in pancreatic cancer. For the first time, we found that combination therapy targeting the IL-6 and IL-8 signaling pathway significantly inhibited cell viability, colony-forming activity and cell migration of PDAC cells. Currently the main methods regarding experimental design to inhibit IL-6 and IL-8 signaling pathways simultaneously are antibodies,
siRNA and antisense transfection $(6,9)$. The main problem associated with such joint inhibition of the IL-6 and IL-8 signaling pathway is that the side-effects are not clear. The drugs bazedoxifene, reparixin and SCH527123 used in our study have been certified as clinically safe and the prospect for their further clinical application may be relatively better. We discovered for the first time that the combination of bazedoxifene with reparixin or SCH527123 is superior to monotherapy in inhibiting cell viability, colony-forming activity and cell migration in TNBC and PDAC cells. We also found that the combination of bazedoxifene with SCH527123 seemed to be more effective against TNBC than the combination of bazedoxifene with reparixin. Novel drug combinations of bazedoxifene and reparixin, as well as bazedoxifene and SCH527123 may provide more effective treatments for TNBC and PDAC with reduced side-effects.

\section{Acknowledgements}

The Authors wish to thank Dr. Rich Eckert at the Department of Biochemistry and Molecular Biology at The University of Maryland (Baltimore, MD, USA) for providing the microscope used for cell migration analysis. 


\section{References}

1 Siegel RL, Miller KD and Jemal A: Cancer statistics, 2018. CA Cancer J Clin 68: 7-30, 2018.

2 Siegel RL, Miller KD and Jemal A: Cancer Statistics, 2017. CA Cancer J Clin 67: 7-30, 2017.

3 Ovcaricek T, Frkovic SG, Matos E, Mozina B and Borstnar S: Triple negative breast cancer-prognostic factors and survival. Radiol Oncol 45: 46-52, 2011.

4 Sharma P: Biology and management of patients with triplenegative breast cancer. Oncologist 21: 1050-1062, 2016

5 Hartman ZC, Poage GM, den Hollander P, Tsimelzon A, Hill J, Panupinthu N, Zhang Y, Mazumdar A, Hilsenbeck SG, Mills GB and Brown PH: Growth of triple-negative breast cancer cells relies upon coordinate autocrine expression of the proinflammatory cytokines IL-6 and IL-8. Cancer Res 73: 3470-3480, 2013.

6 Shi Z, Yang WM, Chen LP, Yang DH, Zhou Q, Zhu J, Chen JJ, Huang RC, Chen ZS and Huang RP: Enhanced chemosensitization in multidrug-resistant human breast cancer cells by inhibition of IL- 6 and IL-8 production. Breast Cancer Res Treat 135: 737-747, 2012.

7 Wigmore SJ, Fearon KC, Sangster K, Maingay JP, Garden OJ and Ross JA: Cytokine regulation of constitutive production of interleukin- 8 and- 6 by human pancreatic cancer cell lines and serum cytokine concentrations in patients with pancreatic cancer. Int J Oncol 21: 881-886, 2002.

8 Singh JK, Simoes BM, Howell SJ, Farnie G and Clarke RB: Recent advances reveal IL- 8 signaling as a potential key to targeting breast cancer stem cells. Breast Cancer Res 15: 210, 2013.

9 Lin Y, Huang R, Chen L, Li S, Shi Q, Jordan C and Huang RP: Identification of interleukin- 8 as estrogen receptor-regulated factor involved in breast cancer invasion and angiogenesis by protein arrays. Int J Cancer 109: 507-515, 2004.

10 Dethlefsen C, Hojfeldt G and Hojman P: The role of intratumoral and systemic IL-6 in breast cancer. Breast Cancer Res Treat 138: 657-664, 2013.

11 Pop VV, Seicean A, Lupan I, Samasca G and Burz CC: IL-6 roles-molecular pathway and clinical implication in pancreatic cancer-A systemic review. Immunol Lett 181: 45-50, 2017.

12 Goumas FA, Holmer R, Egberts JH, Gontarewicz A, Heneweer C, Geisen U, Hauser C, Mende MM, Legler K, Rocken C, Becker T, Waetzig GH, Rose-John S and Kalthoff H: Inhibition of IL-6 signaling significantly reduces primary tumor growth and recurrencies in orthotopic xenograft models of pancreatic cancer. Int J Cancer 137: 1035-1046, 2015.

13 Kuwada Y, Sasaki T, Morinaka K, Kitadai Y, Mukaida N and Chayama K: Potential involvement of IL-8 and its receptors in the invasiveness of pancreatic cancer cells. Int J Oncol 22: 765$771,2003$.

14 Hussain F, Wang J, Ahmed R, Guest SK, Lam EW, Stamp G and El-Bahrawy M: The expression of IL- 8 and IL-8 receptors in pancreatic adenocarcinomas and pancreatic neuroendocrine tumours. Cytokine 49: 134-140, 2010.

15 Chen L, Fan J, Chen H, Meng Z, Chen Z, Wang P and Liu L: The IL-8/CXCR 1 axis is associated with cancer stem cell-like properties and correlates with clinical prognosis in human pancreatic cancer cases. Sci Rep 4: 5911, 2014.

16 Chen Y, Shi M, Yu GZ, Qin XR, Jin G, Chen P and Zhu MH: Interleukin-8, a promising predictor for prognosis of pancreatic cancer. World J Gastroenterol 18: 1123-1129, 2012.
17 de Villiers TJ, Chines AA, Palacios S, Lips P, Sawicki AZ, Levine AB, Codreanu C, Kelepouris N and Brown JP: Safety and tolerability of bazedoxifene in postmenopausal women with osteoporosis: Results of a 5-year, randomized, placebocontrolled phase 3 trial. Osteoporos Int 22: 567-576, 2011.

$18 \mathrm{Li} \mathrm{H}$, Xiao H, Lin L, Jou D, Kumari V, Lin J and Li C: Drug design targeting protein-protein interactions (PPIs) using multiple ligand simultaneous docking (MLSD) and drug repositioning: discovery of raloxifene and bazedoxifene as novel inhibitors of IL-6/GP130 interface. J Med Chem 57: 632-641, 2014.

19 Opfermann P, Derhaschnig U, Felli A, Wenisch J, Santer D, Zuckermann A, Dworschak M, Jilma B and Steinlechner B: A pilot study on reparixin, a CXCR $1 / 2$ antagonist, to assess safety and efficacy in attenuating ischaemia-reperfusion injury and inflammation after on-pump coronary artery bypass graft surgery. Clin Exp Immunol 180: 131-142, 2015.

20 Ginestier C, Liu S, Diebel ME, Korkaya H, Luo M, Brown M, Wicinski J, Cabaud O, Charafe-Jauffret E, Birnbaum D, Guan JL, Dontu G and Wicha MS: CXCR1 blockade selectively targets human breast cancer stem cells in vitro and in xenografts. J Clin Invest 120: 485-497, 2010.

21 Liotti F, De Pizzol M, Allegretti M, Prevete N and Melillo RM: Multiple anti-tumor effects of Reparixin on thyroid cancer. Oncotarget 8: 35946-35961, 2017.

22 Singh S, Sadanandam A, Nannuru KC, Varney ML, Mayer-Ezell $\mathrm{R}$, Bond $\mathrm{R}$ and Singh RK: Small-molecule antagonists for CXCR2 and CXCR1 inhibit human melanoma growth by decreasing tumor cell proliferation, survival, and angiogenesis. Clin Cancer Res 15: 2380-2386, 2009.

23 Wang Y, Liu J, Jiang Q, Deng J, Xu F, Chen X, Cheng F, Zhang Y, Yao Y, Xia Z, Xu X, Su X, Huang M, Dai L, Yang Y, Zhang S, Yu D, Zhao RC, Wei Y and Deng H: Human adipose-derived mesenchymal stem cell-secreted CXCL1 and CXCL8 facilitate breast tumor growth by promoting angiogenesis. Stem Cells 35: 2060-2070, 2017.

24 Ning Y, Labonte MJ, Zhang W, Bohanes PO, Gerger A, Yang D, Benhaim L, Paez D, Rosenberg DO, Nagulapalli VK, Louie SG, Petasis NA, Ladner RD and Lenz HJ: The CXCR2 antagonist, SCH-527123, shows antitumor activity and sensitizes cells to oxaliplatin in preclinical colon cancer models. Mol Cancer Ther 11: 1353-1364, 2012.

$25 \mathrm{Fu}$ S, Chen X, Lin HJ and Lin J: Inhibition of interleukin 8/CX$\mathrm{C}$ chemokine receptor $1, / 2$ signaling reduces malignant features in human pancreatic cancer cells. Int J Oncol 53: 349-357, 2018.

26 Chou TC: Theoretical basis, experimental design, and computerized simulation of synergism and antagonism in drug combination studies. Pharmacol Rev 58: 621-681, 2006.

27 Jayatilaka H, Tyle P, Chen JJ, Kwak M, Ju J, Kim HJ, Lee J, Wu PH, Gilkes DM, Fan R and Wirtz D: Synergistic IL-6 and IL-8 paracrine signalling pathway infers a strategy to inhibit tumour cell migration. Nat Commun 8: 15584, 2017.

28 Heo TH, Wahler J and Suh N: Potential therapeutic implications of IL-6/IL-6R/gp130-targeting agents in breast cancer. Oncotarget 7: 15460-15473, 2016.

29 Conze D, Weiss L, Regen PS, Bhushan A, Weaver D, Johnson P and Rincon M: Autocrine production of interleukin 6 causes multidrug resistance in breast cancer cells. Cancer Res 61: 8851-8858, 2001.

$30 \mathrm{Wu}$ X, Cao Y, Xiao H, Li C and Lin J: Bazedoxifene as a novel GP130 inhibitor for pancreatic cancer therapy. Mol Cancer Ther 15: 2609-2619, 2016. 
31 Xiao H, Bid HK, Chen X, Wu X, Wei J, Bian Y, Zhao C, Li H, $\mathrm{Li} \mathrm{C}$ and Lin $\mathrm{J}$ : Repositioning Bazedoxifene as a novel IL6/GP130 signaling antagonist for human rhabdomyosarcoma therapy. Plos One 12: e180297, 2017.

32 Chen X, Wei J, Li C, Pierson CR, Finlay JL and Lin J: Blocking interleukin-6 signaling inhibits cell viability/proliferation, glycolysis, and colony-forming activity of human medulloblastoma cells. Int J Oncol 52: 571-578, 2018.

33 Wardell SE, Nelson ER, Chao CA and McDonnell DP: Bazedoxifene exhibits antiestrogenic activity in animal models of tamoxifen-resistant breast cancer: implications for treatment of advanced disease. Clin Cancer Res 19: 2420-2431, 2013.

34 Lewis-Wambi JS, Kim H, Curpan R, Grigg R, Sarker MA and Jordan VC: The selective estrogen receptor modulator bazedoxifene inhibits hormone-independent breast cancer cell growth and down-regulates estrogen receptor alpha and cyclin D1. Mol Pharmacol 80: 610-620, 2011.

35 Freund A, Chauveau C, Brouillet JP, Lucas A, Lacroix M, Licznar A, Vignon F and Lazennec G: IL-8 expression and its possible relationship with estrogen-receptor-negative status of breast cancer cells. Oncogene 22: 256-265, 2003.

36 Singh JK, Simoes BM, Clarke RB and Bundred NJ: Targeting IL-8 signalling to inhibit breast cancer stem cell activity. Expert Opin Ther Targets 17: 1235-1241, 2013.
37 Schott AF, Goldstein L, Cristofanilli M, Ruffini PA, McCanna S, Reuben JM, Perez R, Kato G and Wicha MS: Phase Ib pilot study to evaluate reparixin in combination with weekly paclitaxel in patients with HER-2 negative metastatic breast cancer (MBC). Clin Cancer Res 23: 5358-5365, 2017.

38 Nair P, Gaga M, Zervas E, Alagha K, Hargreave FE, O'Byrne PM, Stryszak P, Gann L, Sadeh J and Chanez P: Safety and efficacy of a CXCR2 antagonist in patients with severe asthma and sputum neutrophils: A randomized, placebo-controlled clinical trial. Clin Exp Allergy 42: 1097-1103, 2012.

39 Poage GM, Hartman ZC and Brown PH: Revealing targeted therapeutic opportunities in triple-negative breast cancers: A new strategy. Cell Cycle 12: 2705-2706, 2013.

40 Skolekova S, Matuskova M, Bohac M, Toro L, Durinikova E, Tyciakova S, Demkova L, Gursky J and Kucerova L: Cisplatininduced mesenchymal stromal cells-mediated mechanism contributing to decreased antitumor effect in breast cancer cells. Cell Commun Signal 14: 4, 2016.

Received September 18, 2018

Revised September 25, 2018

Accepted October 2, 2018 